\title{
Neoadjuvant Radiotherapy of Early-Stage and Locally Advanced Breast Cancer: Review of the Literature
}

\author{
Abdelhak Maghous ${ }^{1 *}$, El Amin Marnouche ${ }^{1}$, Noha Zaghba ${ }^{1}$, Khalid Andaloussi ${ }^{1}$, Mohamed Elmarjany ${ }^{1}$, Khalid Hadadi ${ }^{1}$, Hassan Sifat ${ }^{1}$, Rahali Driss Moussaoui $^{2}$ \\ and Hamid Mansouri ${ }^{1}$ \\ ${ }^{1}$ Department of Radiotherapy, Mohammed V Military Teaching Hospital, Mohammed V University in Rabat, Morocco \\ ${ }^{2}$ Department of Gynecology Obstetric, Mohammed V Military Teaching Hospital, Mohammed V University in Rabat, Morocco .

\begin{abstract}
Breast cancer is the most frequently diagnosed cancer type among women worldwide. The treatment of breast cancer is multimodal and the current standard approach is surgery followed by adjuvant radiotherapy (RT) based on prognostic scores. Neoadjuvant chemotherapy and RT delivered in advance of surgery are used in some early stage and locally advanced cases, may offer an advantage over adjuvant therapy related to the potential benefits in the pathologic response and survival outcomes based the hypothesis of Abscopal effect.
\end{abstract}

Keywords: Breast cancer; Neoadjuvant radiotherapy; Concurrent chemo radiotherapy; Abscopal effect

Abbreviations: RT: Radiotherapy or Radiation Therapy; CCRT: Concurrent Chemo Radiotherapy; PCR: Pathologic Complete Response; DFS: Disease-Free Survival; OS: Overall Survival; BCT: Breast Conserving Therapy; SEER: Surveillance, Epidemiology, and End Results; LABC Locally Advanced Breast Cancer; 5-FU: 5-fluorouracil; FEC-D: 5-Fluorouracil, Epirubicin, and Cyclophosphamide - Docetaxel

\section{Introduction}

Breast cancer is the most frequently diagnosed cancer type among women worldwide. This malignancy is managed with a multidisciplinary approach involving surgery, radiation therapy, hormonal therapy and chemotherapy, which has been associated with a reduction in breast cancer mortality [1]. The selection and sequencing of the different treatment are based on patient and tumor variables, including prognostic scores and axillary lymph nodes or distant metastases. The current standard treatment approach is surgery, if technically feasible, followed by adjuvant radiotherapy (RT) in all patients undergoing breast conservation, and postmastectomy in patients with T3 or T4 tumors, or with four or more positive axillary nodes [2-5].

Neoadjuvant chemotherapy before surgery for breast cancer was introduced for women with locally advanced disease to debulk initially inoperable tumors, and later extended toward enhancing the likelihood of breast conservation. Radiation therapy delivered in advance of surgery would reduce delays between surgery and radiation, and may therefore offer an advantage over adjuvant radiotherapy which had confirmed through a number of large randomized phase III trials in a number of other cancer sites, especially rectal cancer [6,7]. However, limited prospective data exist on this therapeutic approach in the setting of breast cancer.

Concurrent Chemo Radiotherapy (CCRT) has the potential to offer patients the combined benefits in the pathologic complete response (PCR) rate and in local and distant disease control. In exploring the role of CCRT in breast cancer, PCR was higher and was associated with better disease-free survival (DFS) and overall survival (OS). However, it seems to be associated with a high rate of toxicities with certain chemotherapy regimens and schedule [8-14]. On the other hand, CCRT limits the duration of treatment and the required hospital visits, without compromising quality of life [15]. Therefore, a prospective randomized clinical trial is warranted to evaluate the optimal chemotherapy regimen, its dose and administration schedule, to better minimize treatment-related toxicity, improved the pathologic response and determine its impact on survival outcome.

The present review describes the hypothesis that motivated outcomes of neoadjuvant RT compared with standard-of-care adjuvant RT, the results of the long-term effects of completed neoadjuvant RT trials in patients with early-stage and locally advanced breast cancer and addressing the use of CCRT in the preoperative setting.

\section{Abscopal Effects of Radiation Therapy}

The term abscopal was defined by Mole in 1953 as a tumor event occurring "at a distance from the irradiated volume but within the same organism" [16]. In oncology, the abscopal effect refers to distant tumor regression after localized irradiation. Otherwise, is believed that arise from the capacity of local RT to elicit systemic immune effects to control unirradiated tumor through several mechanisms.

Localized radiation induces cell stress and immunogenic cell death, which then induced the release of a wealth of previously endogenous damage-associated molecular patterns [17]. RT has also been shown to stimulate tumor cell release of cytokines as a response to ionizing irradiation. Cytokines are thought to elicit augmented tumor surveillance, inhibit tumor growth, and have direct tumoricidal properties [18]. Thus, it is believable that neoadjuvant radiation to the large bulk tumor can activate robust antitumor specific immune responses, which is absent after postoperative RT to the tumor bed, according to recent studies focused to relation between radiation and immune system $[19,20]$. This synergy stems come from the fact that RT potentially converting the tumor into a patient-specific in situ vaccine capable of re-educating the immune system to recognize and reject cancer $[21,22]$. That finding may help eradicate subclinical disease in the opposite breast as well as distant micro metastasis and possibly leading to an immune memory that vaccinates against future tumors [21].

*Corresponding author: Abdelhak Maghous, Department of Radiotherapy, Mohammed V Military Teaching Hospital, Mohammed V University in Rabat, Morocco, Tel: +212661099339; E-mail:magabdelhak@gmail.com

Received March 02, 2018; Accepted March 05, 2018; Published March 08, 2018

Citation: Maghous A, Marnouche EA, Zaghba N, Andalouss Ki, Elmarjany M, et al. (2018) Neoadjuvant Radiotherapy of Early-Stage and Locally Advanced Breast Cancer: Review of the Literature. J Nucl Med Radiat Ther 9: 357. doi: 10.4172/2155-9619.1000357

Copyright: () 2018 Maghous A, et al . This is an open-access article distributed under the terms of the Creative Commons Attribution License, which permits unrestricted use, distribution, and reproduction in any medium, provided the original author and source are credited. 
The abscopal effect motivated the analysis of long-term outcomes of neoadjuvant radiation compared with adjuvant one in patients with breast cancer. Based on this promising result and the hypothesis that the immune system is a major process in mediating the abscopal effect, several clinical trials investigating the combination of immunotherapy and radiation in breast cancer patients are actively ongoing [23]

\section{Neoadjuvant Radiotherapy of Early-Stage Breast Cancer}

Currently, patients with early-stage breast cancer may undergo primary breast surgery (lumpectomy or mastectomy) and regional lymph node excision with or without radiation therapy (RT). Adjuvant RT is performed following Breast Conserving Therapy (BCT) or mastectomy in patients who are considered to be at greater risk of recurrence [24]. Neoadjuvant chemotherapy was extended to early breast cancer, mainly to allow BCT more feasible, and is now widely used, particularly for large tumors $[25,26]$.

However, data about adjuvant RT in the setting of early-stage breast cancer is limited. A prospective nonrandomized, German study was the first who had identified a survival advantage for neoadjuvant RT \pm chemotherapy compared with adjuvant therapy in T2 tumors. Neoadjuvant RT achieved in this study a PCR rate of $29.2 \%$ and a statistically significantly better DFS and OS [27]. Recently, the analysis of the Surveillance, Epidemiology, and End Results (SEER) database provided by the National Institutes of Health about 250,195 female patients with early-stage breast cancer who received RT before or after surgery. The results suggest that RT before surgery may significantly reduce the incidence of second primary tumors and improve DFS without decreasing OS rates, especially for estrogen receptor-positive patients with early-stage breast cancer [28]. However, these findings still retrospective analysis without systemic treatment records, and no data regarding selection criteria for neoadjuvant RT. Further prospective randomized clinical trial, with correlative studies done to identify mechanisms of potential benefits of neoadjuvant RT for earlystage breast cancer are warranted.

\section{Neoadjuvant Radiotherapy of Locally Advanced Breast Cancer}

Locally advanced breast cancer (LABC) is a subset of breast cancer characterized by the most advanced breast tumors in the absence of distant metastasis. It was recognized that multimodal therapy including; surgery, chemotherapy and radiotherapy can significantly improve outcomes in this select group of patients [29]. Neo-adjuvant chemotherapy integrated into this multimodality program is the conventional treatment in LABC [30,31]. Primary systemic over adjuvant therapy has the advantages of in vivo measurement of response to therapy, increased the chances of successful BCT as well as the reduction of microscopic neoplastic dissemination [31-33]

The benefit of CCRT in the PCR rate and in local and distant disease control has made that modality the standard of care in a number of malignancies. However, only limited published data exist for the use of neoadjuvant CCRT in LABC. The most common small phase II reports were looking at 5-fluorouracil (5-FU) or capecitabine based radiosensitizing agent, in inoperable or inflammatory breast cancer and who progress on first line anthracycline-based chemotherapy [34-36]. Although those agents are generally not considered the most active in breast cancer, current retrospective studies in highly selected patients, most using 5-FU as the radiosensitizing agent, have been shown some benefit in the PCR rate and in OS without added toxicity [12,13].
Other phase II trials showed a benefit from neoadjuvant CCRT using paclitaxel as the radiosensitizer. Unfortunately, it seems to be associated with a high rate of toxicities [12,37].

In the other hand Formenti et al. [8] has concluded that toxicities can be considerably reduced if paclitaxel is administered twice weekly at $30 \mathrm{mg} / \mathrm{m}^{2}$ as an alternative of weekly at $80 \mathrm{mg} / \mathrm{m}^{2}$ or as a continuous infusion at $20-30 \mathrm{mg} / \mathrm{m}^{2}$ daily. Moreover, update data demonstrated that pathological response was higher and was associated with better disease-free survival (DFS) and overall survival OS when compared with non-responders within the same treatment cohort [8].

Recent prospective phase II trial evaluated administration of every-three-weeks 5-fluorouracil, epirubicin, and cyclophosphamide (FEC) in standard adjuvant dosing, followed by weekly docetaxel at $35 \mathrm{mg} / \mathrm{m}^{2}$ through 6 weeks of concurrent preoperatively radiation. Brackstone et al. [8] in this first clinical trial evaluating concurrent neoadjuvant CCRT with a taxane as part of a modern chemotherapy regimen (FEC-D), support the same findings of the Formenti group. He suggests that this regimen significantly improved the PCR rate in LABC patients; however, the benefit shown in DFS and OS at 3 years was not statistically significant when compared with a propensity-matched control group. In term of toxicities docetaxel seems to be associated with a high rate of pneumonitis which resolved acutely and did not lead to long-term impairment.

\section{Conclusions}

Radiation therapy delivered in advance of surgery would reduce delays between surgery and radiation, and may therefore represent a favorable option. Ambitious current prospective data analyzing neoadjuvant RT of early-stage and locally advanced breast cancer demonstrated a real benefit in the pathologic response and potential survival advantages rates. However, it seems to be associated with a high rate of toxicities when prescribed concurrently with certain chemotherapy regimens, dose and administration schedule. Promising results was shown with concurrent twice-weekly paclitaxel or decetaxel, even with high pneumonitis using docetaxel. Therefore a future large, prospective randomized clinical trial should be undertaken before recommending taxanes concurrently with locoregional RT.

\section{Competing Interests}

We (authors) declare that we have no conflict of interest.

\section{Acknowledgement}

All the authors are thankful for providing the necessary to improve the manuscript.

\section{References}

1. Kesson EM, Allardice GM, George WD (2012) Effects of multidisciplinary team working on breast cancer survival: Retrospective, comparative, interventiona cohort study of 13722 women. BMJ 344: e2718.

2. Recht A, Edge SB, Solin LJ (2001) Postmastectomy radiotherapy: Clinical practice guidelines of the American Society of Clinical Oncology. J Clin Oncol 19: $1539-1569$.

3. Eifel P, Axelson JA, Costa J, Crowley J, Curran WJ Jr, et al. (2001) National Institutes of Health Consensus Development Conference statement: Adjuvant therapy for breast cancer. November 1-3, 2000, J Natl Cancer Inst Monographs 30: 5-15.

4. Carlson RW, Allred DC, Anderson BO (2012) Metastatic breast cancer, version 1.2012: Featured updates to the NCCN guidelines. J Natl Compr Canc Netw 10: $821-829$.

5. Truong PT, Olivotto IA, Whelan TJ (2004) Clinical practice guidelines fo the care and treatment of breast cancer: 16 . Locoregional post-mastectomy radiotherapy. CMAJ 170: 1263-1273. 
Citation: Maghous A, Marnouche EA, Zaghba N, Andalouss Ki, Elmarjany M, et al. (2018) Neoadjuvant Radiotherapy of Early-Stage and Locally Advanced Breast Cancer: Review of the Literature. J Nucl Med Radiat Ther 9: 357. doi: 10.4172/2155-9619.1000357

6. Valentini V, van Stiphout RG, Lammering G (2011) Nomograms for predicting local recurrence, distant metastases, and overall survival for patients with locally advanced rectal cancer on the basis of European randomized clinical trials. J Clin Oncol 29: 3163-3172.

7. Popek S, Tsikitis VL (2011) Neoadjuvant vs adjuvant pelvic radiotherapy for locally advanced rectal cancer: Which is superior? World J Gastroenterol 17: $848-854$

8. Formenti SC, Volm M, Skinner KA (2003) Preoperative twice-weekly paclitaxel with concurrent radiation therapy followed by surgery and postoperative doxorubicin-based chemotherapy in locally advanced breast cancer: A phase I/ II trial. J Clin Oncol 21: 864-870.

9. Adams S, Chakravarthy AB, Donach M (2010) Preoperative concurrent paclitaxel-radiation in locally advanced breast cancer: Pathologic response correlates with five-year overall survival. Breast Cancer Res Treat 124: 723732.

10. Brackstone M, Palma D, Tuck AB, Scott L, Potvin K et al. (2017) Concurrent neoadjuvant chemotherapy and radiation therapy in locally advanced breast cancer. Int J Radiat Oncol Biol Phys 99: 769-776.

11. Kao J, Conzen SD, Jaskowiak NT (2005) Concomitant radiation therapy and paclitaxel for unresectable locally advanced breast cancer: Results from two consecutive phase i/ii trials. Int J Radiat Oncol Biol Phys 61: 1045-1053.

12. Bollet MA, Belin L, Reyal F (2012) Preoperative radio-chemotherapy in early breast cancer patients: Long-term results of a phase II trial. Radiother Oncol 102: 82-88.

13. Alvarado-Miranda A, Arrieta O, Gamboa-Vignolle C (2009) Concurrent chemoradiotherapy following neoadjuvant chemotherapy in locally advanced breast cancer. Radiat Oncol 4: 24.

14. Chow TL, Louie AV, Palma DA (2014) Radiation-induced lung injury after concurrent neoadjuvant chemoradiotherapy for locally advanced breast cancer. Acta Oncol 53: 697-701.

15. Macquart-Moulin G, Viens P, Genre D (1999) Concomitant chemoradiotherapy for patients with nonmetastatic breast carcinoma: Side effects, quality of life, and organization. Cancer 85: 2190-2199.

16. Mole R (1953) Whole body irradiation-radiobiology or medicine? $\mathrm{Br}$ J Radio 26: $234-241$

17. Vatner RE, Cooper BT, Vanpouille-Box C, Demaria S, Formenti SC (2014) Combinations of immunotherapy and radiation in cancer therapy. Front Oncol 4: 325 .

18. Kaminski JM, Shinohara E, Summers JB, Niermann KJ, Morimoto A, et al. (2005) The controversial abscopal effect. Cancer Treat Rev 3: 159-172.

19. Burnette B, Weichselbaum RR (2013) Radiation as an immune modulator. Semin Radiat Oncol 23: 273-280.

20. Multhoff G, Radons J (2012) Radiation, inflammation, and immune responses in cancer. Front Oncol 2: 58.

21. Formenti SC, Demaria S (2012) Radiation therapy to convert the tumor into an in situ vaccine. Int J Radiat Oncol 84: 879-80.
22. Poleszczuk JT, Luddy KA, Prokopiou S, Robertson-Tessi M, Moros EG, et al (2016) Abscopal benefits of localized radiotherapy depend on activated T-cell trafficking and distribution between metastatic lesions. Cancer Res 76: 10091018.

23. Hu Zl, McArthur HL, Ho AY (2017) The Abscopal Effect of Radiation Therapy: What is it and how can we use it in breast cancer? Curr Breast Cancer Rep 9: 45-51.

24. Gradishar WJ, Anderson BO, Balassanian R, Blair SL, Burstein HJ, et al (2015) NCCN Guidelines Insights Breast Cancer, Version 1.2016. J Natl Compr Canc Netw 13: 1475-1485.

25. Clough KB, Acosta-Marín V, Nos C, Alran S, Rouanet P, et al. (2015) Rates of neoadjuvant chemotherapy and oncoplastic surgery for breast cancer surgery: A french national survey. Ann Surg Oncol 11: 3504-3511.

26. Vugts G, Maaskant-Braat AJ, Nieuwenhuijzen GA, Roumen RM, Luiten EJ, et al. (2016) Patterns of care in the administration of neoadjuvant chemotherapy for breast cancer. A population-based study. Breast J 22: 316-321.

27. Roth SL, Audretsch W, Bojar H, Lang I, Willers R, et al. (2010) Retrospective study of neoadjuvant versus adjuvant radiochemotherapy in locally advanced noninflammatory breast cancer: Survival advantage in cT2 category by neoadjuvant radiochemotherapy, Strahlenther Onkol 186: 299-306.

28. Poleszczuk J, Luddy K, Chen L, Lee JK, Harrison LB, et al. (2017) Neoadjuvant radiotherapy of early-stage breast cancer and long-term diseasefree survival. Breast Cancer Res 19: 75

29. Giordano SH (2003) Update on locally advanced breast cancer. Oncologist 8 : 521-530.

30. Specht J, Gralow JR (2009) Neoadjuvant chemotherapy for locally advanced breast cancer. Semin Radiat Oncol 19: 222-228.

31. Liu SV, Melstrom L, Yao K, Russell CA, Sener SF (2010) Neoadjuvant therapy for breast cancer. J Surg Oncol 101: 283-291.

32. Mamounas EP (2003) Neoadjuvant chemotherapy for operable breast cancer: Is this the future? Clin Breast Cancer 4: s10-19.

33. Sinclair S, Swain SM (2010) Primary systemic chemotherapy for inflammatory breast cancer. Cancer 116: s2821-2828.

34. Ren H, Wang Qi, Yaoxiong Y (2006) Preoperative chemoradiotherapy for inflammatory breast cancer. Chin J Clin Oncol 3: 130-133.

35. Gaui MF, Amorim G, Arcuri RA, Pereira G, Moreira D, et al. (2007) A phase II study of second-line neoadjuvant chemotherapy with capecitabine and radiation therapy for anthracycline-resistant locally advanced breast cancer Am J Clin Oncol 30: 78-81.

36. Touboul E, Lefranc JP, Blondon J, Buffat L, Deniaud E, et al. (1997) Primary chemotherapy and preoperative irradiation for patients with stage II larger than $3 \mathrm{~cm}$ or locally advanced non-inflammatory breast cancer. Radiother Oncol 42 219-229.

37. Skinner KA Silberman H, Florentine B, Lomis TJ, Corso F, et al. (2000) Preoperative paclitaxel and radiotherapy for locally advanced breast cancer: Surgical aspects. Ann Surg Oncol 7: 145-149. 